DOI 10.4171/JEMS/369

Saugata Basu $\cdot$ Andrei Gabrielov · Nicolai Vorobjov

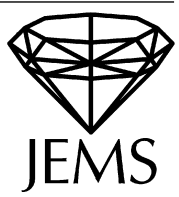

\title{
Semi-monotone sets
}

Received May 5, 2010

\begin{abstract}
A coordinate cone in $\mathbb{R}^{n}$ is an intersection of some coordinate hyperplanes and open coordinate half-spaces. A semi-monotone set is an open bounded subset of $\mathbb{R}^{n}$, definable in an ominimal structure over the reals, such that its intersection with any translation of any coordinate cone is connected. This notion can be viewed as a generalization of convexity. Semi-monotone sets have a number of interesting geometric and combinatorial properties. The main result of the paper is that every semi-monotone set is a topological regular cell.
\end{abstract}

\section{Introduction}

It is well known that in o-minimal geometry, definable sets that are locally closed are easier to handle than arbitrary definable sets. A typical example of this phenomenon can be seen in the well-studied problem of obtaining tight upper bounds on topological invariants such as the Betti numbers of semi-algebraic or semi-Pfaffian sets in terms of the complexity of formulae defining them. Certain standard techniques from algebraic topology (for example, inequalities stemming from the Mayer-Vietoris exact sequence) are directly applicable only in the case of locally closed definable sets. Definable sets which are not locally closed are comparatively more difficult to analyze. In order to overcome this difficulty, Gabrielov and Vorobjov [3] suggested a construction which, given a definable set $S$ in an o-minimal structure over the reals, produced an explicit family of definable compact sets converging to $S$. Under a certain technical condition (called "separability") they proved that the approximating compact sets are homotopy equivalent to $S$. The separability condition is automatically satisfied in many cases of interest-such as when $S$ is described by equations and inequalities with continuous definable functions.

However, the property of separability is not preserved under taking images of definable maps, and this restricts the applicability of this construction. It was conjectured in [3] that the crucial property of the approximating family (homotopy equivalence to $S$ ) remains true even without the separability hypothesis. Proving this conjecture seems to be

S. Basu, A. Gabrielov: Department of Mathematics, Purdue University, West Lafayette, IN 47907, USA; e-mail: sbasu@math.purdue.edu, agabriel@math.purdue.edu

N. Vorobjov: Department of Computer Science, University of Bath,

Bath, BA2 7AY, England, UK; e-mail: nnv@cs.bath.ac.uk

Mathematics Subject Classification (2010): Primary 14P15; Secondary 57Q99 
a rather difficult problem at present. One of the authors of the current paper (Gabrielov) has outlined a research program whose completion would lead (amongst other things) to a proof of the conjecture. The goal of the program is a "triangulation" of an increasing definable family of compact sets. More precisely, the goal is to prove that given any increasing definable family of compact sets converging to a definable set $S \subset \mathbb{R}^{n}$, there exists a definable triangulation of $\mathbb{R}^{n}$ such that inside each open simplex of this triangulation the increasing definable family belongs to a finite list of combinatorial types. Such a triangulation should be considered as being compatible with the given increasing family (thus generalizing the standard notion of definable triangulations compatible with a given definable set). The homotopy equivalence conjecture will then follow from this triangulation.

One of the key steps in Gabrielov's program is to prove the existence of a regular triangulation of the graph of a definable function. More precisely, there is the following conjecture.

Conjecture 0.1. Let $f: K \rightarrow \mathbb{R}$ be a definable function on a compact definable set $K \subset \mathbb{R}^{m}$. Then there exists a definable triangulation of $K$ such that, for each $n \leq \operatorname{dim} K$ and for each open $n$-simplex $\Delta$ of the triangulation,

1. the graph $\Gamma:=\{(\mathbf{x}, t) \mid \mathbf{x} \in \Delta, t=f(\mathbf{x})\}$ of the restriction of $f$ to $\Delta$ is a regular $n$-cell (see Definition 2.1);

2. either $f$ is a constant on $\Delta$ or each non-empty level set $\Gamma \cap\{t=\mathrm{const}\}$ is a regular $(n-1)$-cell.

It should be pointed out that Conjecture 0.1 does not follow from results in the literature on the existence of definable triangulations adapted to a given finite family of definable subsets of $\mathbb{R}^{n}$ (such as [7, 2]), since all the proofs use a preparatory linear change of coordinates in order for the given definable sets to be in a good position with respect to coordinate projections. Since we are concerned with the graphs and the level sets of a function, in proving Conjecture 0.1 we are not allowed to make any change of coordinates which involves the last coordinate. Thus, the standard methods of obtaining a definable triangulation using "cylindrical decomposition" are not immediately applicable. In the book [7], van den Dries describes a strong form of cylindrical decomposition in which the cells are defined by functions having a coordinatewise monotonicity property (such cells are called regular in [7]). We show that in fact these cells are not necessarily regular cells in the sense of topology (see Definition 2.1). To prove Conjecture 0.1, we need a sufficiently general class of definable sets which are guaranteed to be topologically regular cells.

In this paper, we introduce a new class of definable sets, which we call semi-monotone sets, and show that a non-empty semi-monotone set in $\mathbb{R}^{n}$ is a regular $n$-cell. A coordinate cone in $\mathbb{R}^{n}$ is an intersection of some coordinate hyperplanes and open coordinate half-spaces. A semi-monotone set is an open bounded subset of $\mathbb{R}^{n}$, definable in an ominimal structure over the reals, such that its intersection with any translation of any coordinate cone is connected. It is obvious that every convex definable bounded open set is semi-monotone. Some non-convex examples as well as counter-examples are shown in Figure 1. 

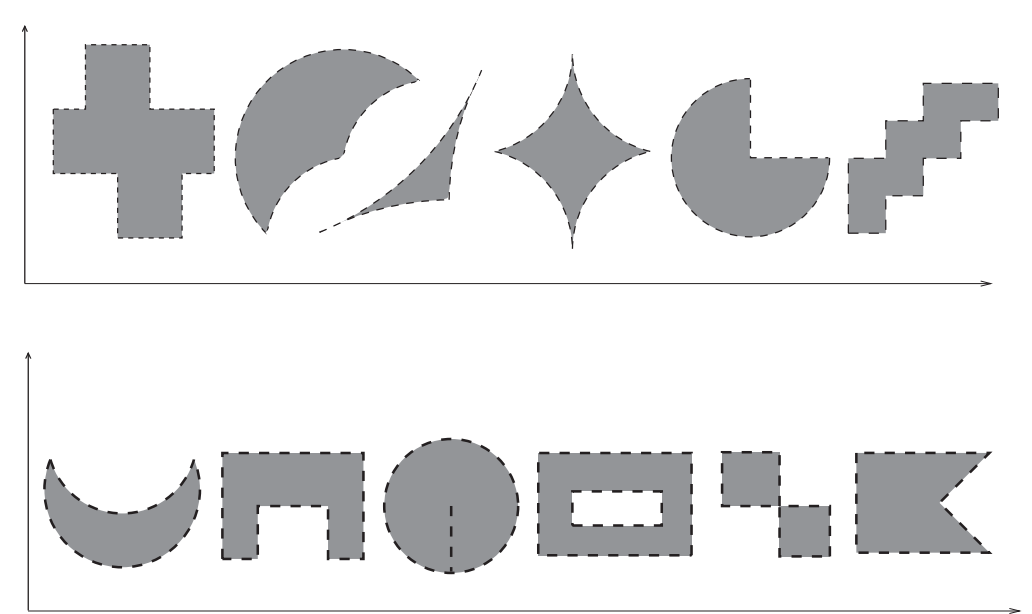

Fig. 1. Top: examples of semi-monotone subsets of the plane. Bottom: examples of open subsets of the plane which are not semi-monotone.

The paper is organized as follows. In Section 1 we define semi-monotone sets and prove necessary and sufficient conditions for an open bounded set to be semi-monotone, which are similar to the properties of cylindrical cells in o-minimal geometry. In particular, it is proved that any semi-monotone set is a "band" between the graphs of two semi-continuous functions which are defined on a semi-monotone set of a smaller dimension and have certain monotonicity properties.

Section 2 contains the proof of the main result, that every semi-monotone set is a regular cell. In Section 3 we prove the regularity in the case of semi-algebraic semi-monotone sets defined over an arbitrary real closed field. In Section 4 we show that cylindrical cells called "regular" in [7] are not necessarily topologically regular.

In Section 5 a concept of a regular Boolean function is introduced. A Boolean function $\psi\left(\xi_{1}, \ldots, \xi_{n}\right)$ in $n$ Boolean variables $\xi_{j} \in\{0,1\}$ is called regular if the result of any sequence of operations $\forall \xi_{j}$ and $\exists \xi_{k}$ applied to $\psi$ does not depend on the order of the operations. To every point $p$ outside a given open bounded set $U$ we assign a Boolean function, taking the value 1 exactly on the octants with vertex $p$ which have a non-empty intersection with $U$ (see Definition 5.7). The main result of Section 5 is that $U$ is semimonotone if and only if the functions assigned to all points outside $U$ are regular.

Section 6 is an appendix containing some known and new facts from PL topology needed in the proof of the main result.

\section{Equivalent definitions of semi-monotone sets}

In what follows we fix an o-minimal structure over $\mathbb{R}$, and consider only sets and maps that are definable in this structure. 
Definition 1.1. Let

$$
X_{j, \sigma, c}:=\left\{\mathbf{x}=\left(x_{1}, \ldots, x_{n}\right) \in \mathbb{R}^{n} \mid x_{j} \sigma c\right\},
$$

for $1 \leq j \leq n, \sigma \in\{<,=,>\}$ and $c \in \mathbb{R}$. An open (possibly empty) bounded set $U \subset \mathbb{R}^{n}$ is called semi-monotone if

$$
U \cap X_{j_{1}, \sigma_{1}, c_{1}} \cap \cdots \cap X_{j_{k}, \sigma_{k}, c_{k}}
$$

is connected for any $0 \leq k \leq n$, any $1 \leq j_{1}<\cdots<j_{k} \leq n$, any $\sigma_{1}, \ldots, \sigma_{k}$ in $\{<,=,>\}$, and any $c_{1}, \ldots, c_{k} \in \mathbb{R}$.

Lemma 1.2. The projection of a semi-monotone set $U$ on any coordinate subspace is a semi-monotone set.

Proof. Let $U^{\prime}$ be the projection of $U$ on the subspace of coordinates $x_{1}, \ldots, x_{m}$ where $m \leq n$. Then any intersection

$$
U^{\prime} \cap X_{j_{1}, \sigma_{1}, c_{1}}^{\prime} \cap \cdots \cap X_{j_{k}, \sigma_{k}, c_{k}}^{\prime},
$$

where $X_{j, \sigma, c}^{\prime}=\left\{\left(x_{1}, \ldots, x_{m}\right) \in \mathbb{R}^{m} \mid x_{j} \sigma c\right\}$ and $1 \leq j_{1}<\cdots<j_{k} \leq m$, is connected as the projection of the connected set

$$
U \cap X_{j_{1}, \sigma_{1}, c_{1}} \cap \cdots \cap X_{j_{k}, \sigma_{k}, c_{k}} .
$$

Theorem 1.3. An open and bounded set $U \subset \mathbb{R}^{n}$ is semi-monotone if and only if both of the following conditions hold:

(S1) its intersection with any line parallel to the $x_{n}$-axis is either empty or an open interval,

(S2) the projections of the sets $U \cap X_{n, \sigma, c}$ to $\mathbb{R}^{n-1}$ along the $x_{n}$-axis are semi-monotone sets in $\mathbb{R}^{n-1}$ for any $\sigma \in\{<,=,>\}$ and $c \in \mathbb{R}$.

Proof. We prove the statement by induction on $n$. For $n=1$ it is obvious. Let $U$ satisfy (S1) and (S2). The set $U$ is connected, otherwise its projection $U^{\prime}$ along the $x_{n}$-axis would be disconnected (a very special case of the Vietoris-Begle theorem, [6]). This would contradict (S2), since $U=U \cap X_{n,<, c}$ for large positive $c$ and hence its projection is connected.

For $j_{k}<n$, the projection of

$$
U \cap X_{j_{1}, \sigma_{1}, c_{1}} \cap \cdots \cap X_{j_{k}, \sigma_{k}, c_{k}}
$$

to $\mathbb{R}^{n-1}$ is equal to

$$
U^{\prime} \cap X_{j_{1}, \sigma_{1}, c_{1}}^{\prime} \cap \cdots \cap X_{j_{k}, \sigma_{k}, c_{k}}^{\prime},
$$

where $X_{j, \sigma, c}^{\prime}=\left\{\mathbf{x}=\left(x_{1}, \ldots, x_{n-1}\right) \in \mathbb{R}^{n-1} \mid x_{j} \sigma c\right\}$. This set is connected by the inductive hypothesis, hence

$$
U \cap X_{j_{1}, \sigma_{1}, c_{1}} \cap \cdots \cap X_{j_{k}, \sigma_{k}, c_{k}}
$$

is connected, by the Vietoris-Begle theorem. 
For $j_{k}=n$, the projection of

$$
U \cap X_{j_{1}, \sigma_{1}, c_{1}} \cap \cdots \cap X_{j_{k}, \sigma_{k}, c_{k}}
$$

to $\mathbb{R}^{n-1}$ is equal to the intersection of the projection of $U \cap X_{n, \sigma_{k}, c_{k}}$ and the set

$$
X_{j_{1}, \sigma_{1}, c_{1}}^{\prime} \cap \cdots \cap X_{j_{k-1}, \sigma_{k-1}, c_{k-1}}^{\prime} .
$$

It is connected due to condition (S2) and the induction hypothesis, hence

$$
U \cap X_{j_{1}, \sigma_{1}, c_{1}} \cap \cdots \cap X_{j_{k}, \sigma_{k}, c_{k}}
$$

is connected, again by the Vietoris-Begle theorem.

Conversely, if $U$ is a semi-monotone set, its intersection with each line parallel to any coordinate axis is connected, i.e., either empty or an open interval. Since all sets

$$
U \cap X_{n, \sigma, c} \cap X_{j_{1}, \sigma_{1}, c_{1}} \cap \cdots \cap X_{j_{k}, \sigma_{k}, c_{k}}
$$

are connected, their projections along the $x_{n}$-axis are connected. This implies that projections of the sets $U \cap X_{n, \sigma, c}$ along the $x_{n}$-axis are semi-monotone sets in $\mathbb{R}^{n-1}$.

Corollary 1.4. (1) If $U \subset \mathbb{R}^{n}$ is a semi-monotone set, then $U \cap X_{j,<, a} \cap X_{j,>, b}$ is a semi-monotone set in $\mathbb{R}^{n}$ for any $1 \leq j \leq n$ and $a, b \in \mathbb{R}$.

(2) If $U \subset \mathbb{R}^{n}$ is a semi-monotone set, then

$$
U \cap X_{j_{1}, \sigma_{1}, c_{1}} \cap \cdots \cap X_{j_{k}, \sigma_{k}, c_{k}}
$$

is semi-monotone for any $0 \leq k \leq n$, any $1 \leq j_{1}<\cdots<j_{k} \leq n$, any $\sigma_{1}, \ldots, \sigma_{k}$ in $\{<,=,>\}$, and any $c_{1}, \ldots, c_{k} \in \mathbb{R}$.

Proof. (1) The statement is obvious for $n=1$. Let $n \geq 2$ and $j<n$. Then the set $U \cap X_{j,<, a} \cap X_{j,>, b}$ satisfies conditions (S1) and (S2), hence it is semi-monotone.

(2) immediately follows from (1).

Corollary 1.5. Any semi-monotone set $U$ is acyclic.

Proof. We prove the statement by induction on $n$. The case $n=1$ is obvious. Applying Theorem 1.3, (S2), to $U=U \cap X_{n,<, c}$ for a large positive $c$ we conclude that the projection $U^{\prime}$ of $U$ along the $x_{n}$-axis is a semi-monotone set, and therefore, by the inductive hypothesis, is acyclic. By (S1), the fibres of this projection map are acyclic, so, since the projection is an open map, the Vietoris-Begle theorem implies that $U$ is also acyclic.

Note that in Theorem 2.2 we will prove a much stronger result.

Definition 1.6. A bounded upper semi-continuous function $f$ defined on a semi-monotone set $U \subset \mathbb{R}^{n}$ is submonotone if, for any $r>\inf _{\mathbf{x} \in U} f(x)$, the set

$$
\{\mathbf{x} \in U \mid f(\mathbf{x})<r\}
$$

is semi-monotone. A function $f$ is supermonotone if $-f$ is submonotone. 
Theorem 1.7. An open and bounded set $U \subset \mathbb{R}^{n}$ is semi-monotone if and only if it satisfies the following conditions. If $U \subset \mathbb{R}^{1}$ then $U$ is an open interval. If $U \subset \mathbb{R}^{n}$, then

$$
U=\left\{(\mathbf{x}, t) \mid \mathbf{x} \in U^{\prime}, f(\mathbf{x})<t<g(\mathbf{x})\right\}
$$

for some functions $f$ and $g$ on a semi-monotone set $U^{\prime} \subset \mathbb{R}^{n-1}$, where $f(\mathbf{x})<g(\mathbf{x})$ for all $\mathbf{x} \in U^{\prime}$, with $f(\mathbf{x})$ being submonotone and $g(\mathbf{x})$ being supermonotone.

Proof. Suppose that $U$ is semi-monotone, and $U^{\prime}$ is the projection of $U$ on the subspace of coordinates $x_{1}, \ldots, x_{n-1}$. By Lemma 1.2, $U^{\prime}$ is a semi-monotone set. According to (S1) of Theorem 1.3, any fibre of the projection map over a point $\mathbf{x} \in U^{\prime}$ is an open interval. Take the lower endpoints of these intervals as values of $f$ and upper endpoints as values of $g$. It follows that

$$
U=\left\{(\mathbf{x}, t) \mid \mathbf{x} \in U^{\prime}, f(\mathbf{x})<t<g(\mathbf{x})\right\} .
$$

The function $f$ is bounded because $U$ is bounded. The function $f$ is upper semicontinuous since otherwise there would exist a sequence $\mathbf{x}^{(i)} \in U^{\prime}$ with $\lim _{i \rightarrow \infty} \mathbf{x}^{(i)}=$ $\mathbf{x}^{(0)} \in U^{\prime}$ such that $\lim _{i \rightarrow \infty} f\left(\mathbf{x}^{(i)}\right)-f\left(\mathbf{x}^{(0)}\right)>\varepsilon$ for some positive $\varepsilon \in \mathbb{R}$. Then the interval with lower endpoint $f\left(\mathbf{x}^{(0)}\right)$ has a point belonging both to $U$ and to the boundary of $U$, which contradicts the openness of $U$.

Let $r>\inf _{\mathbf{x} \in U^{\prime}} f(\mathbf{x})$. The definition of $f$ implies that the set

$$
S_{r}:=\left\{\mathbf{x} \in U^{\prime} \mid f(\mathbf{x})<r\right\}
$$

is the projection of $U \cap X_{n,<, r}$ on the subspace of coordinates $x_{1}, \ldots, x_{n-1}$. According to Corollary 1.4, $U \cap X_{n,<, r}$ is a semi-monotone set, thus, by Lemma 1.2, its projection $S_{r}$ is also semi-monotone. It follows that $f$ is submonotone.

Similarly, the function $g$ is supermonotone.

We have proved that each semi-monotone $U$ satisfies the conditions in the theorem. Now assume that an open and bounded set $U \subset \mathbb{R}^{n}$ satisfies these conditions, in particular, its projection $U^{\prime}$ is semi-monotone. We prove that $U$ is semi-monotone by induction on $n$, the case $n=1$ being trivial.

According to Theorem 1.3, it is sufficient to prove that $U$ satisfies conditions (S1) and (S2). Condition (S1) holds true because every intersection of $U$ with a straight line parallel to the $x_{n}$-axis is an interval $(f(\mathbf{x}), g(\mathbf{x}))$ for some $\mathbf{x} \in U^{\prime}$.

For any $c \in\left(\inf _{\mathbf{x} \in U^{\prime}} f(\mathbf{x}), \sup _{\mathbf{x} \in U^{\prime}} g(\mathbf{x})\right)$ the projection of $U \cap X_{n,<, c}$ on the subspace of coordinates $x_{1}, \ldots, x_{n-1}$ coincides with $\left\{\mathbf{x} \in U^{\prime} \mid f(\mathbf{x})<c\right\}$ and therefore is a semimonotone set. Similarly, the projection of $U \cap X_{n,>, c}$ is a semi-monotone set. By the Vietoris-Begle theorem, both sets $U \cap X_{n,<, c}$ and $U \cap X_{n,>, c}$ are connected.

To establish condition (S2) of Theorem 1.3 it remains to prove that the projection $W$ of $U \cap X_{n,=, c}$ is also a semi-monotone set. We will prove this by showing that any intersection of the kind $W \cap X_{j_{1}, \sigma_{1}, c_{1}} \cap \cdots \cap X_{j_{k}, \sigma_{k}, c_{k}}$ is connected, where $j_{1}<\cdots<$ $j_{k}<n$ and $\sigma_{1}, \ldots, \sigma_{k} \in\{<,=,>\}$. For this, it is enough to prove that any intersection $U \cap X_{n,=, c} \cap X_{j_{1}, \sigma_{1}, c_{1}} \cap \cdots \cap X_{j_{k}, \sigma_{k}, c_{k}}$ is connected. If at least one $\sigma_{i}$ is $=$, then the connectedness follows from the inductive hypothesis, since the conditions in the theorem 
are compatible with the translated coordinate cones $X_{j_{1}, \sigma_{1}, c_{1}} \cap \cdots \cap X_{j_{k}, \sigma_{k}, c_{k}}$. Otherwise, $U \cap X_{j_{1}, \sigma_{1}, c_{1}} \cap \cdots \cap X_{j_{k}, \sigma_{k}, c_{k}}$ is itself a bounded open set in $\mathbb{R}^{n}$ satisfying the conditions of the theorem, and it remains to prove that the intersection of this set with $X_{n,=, c}$ or, without a loss of generality, the intersection $U \cap X_{n,=, c}$, is connected.

Suppose that $U \cap X_{n,=, c}$ is not connected. Every fibre $U \cap X_{n,=, c} \cap X_{n-1,=, t}$ of the projection of $U \cap X_{n,=, c}$ on the $x_{n-1}$-axis is a semi-monotone cell by the inductive hypothesis, hence, by Corollary 1.5, is acyclic. Then the Vietoris-Begle theorem implies that the image of the projection of $U \cap X_{n,=, c}$ is also disconnected, i.e., there is a point $t_{0}$ such that $U \cap X_{n,=, c} \cap X_{n-1,=, t_{0}}=\emptyset$ while both sets $U \cap X_{n,=, c} \cap X_{n-1,<, t_{0}}$ and $U \cap X_{n,=, c} \cap X_{n-1,>, t_{0}}$ are non-empty. Because $U$ is open while the sets $U \cap X_{n,<, c}$ and $U \cap X_{n,>, c}$ are connected, each of them has a non-empty intersection with $X_{n-1,=, t_{0}}$. But this implies that $U \cap X_{n-1,=, t_{0}}$ is not connected, which contradicts what was proved before.

\section{Semi-monotone sets are regular cells}

Any compact definable set in $\mathbb{R}^{n}$ admits a finite triangulation (see, e.g., [7]), in particular is definably homeomorphic to a polyhedron. Any open set in $\mathbb{R}^{n}$ is a polyhedron.

Definition 2.1. A definable set $U$ is called a regular $k$-cell if the pair $(\bar{U}, U)$ is definably homeomorphic to the pair $\left([-1,1]^{k},(-1,1)^{k}\right)$.

In this section we say that a definable set is a closed $n$-ball if it is definably homeomorphic to $[-1,1]^{n}$, is an open $n$-ball if it is definably homeomorphic to $(-1,1)^{n}$, and is an $(n-1)$-sphere if it is definably homeomorphic to $[-1,1]^{n} \backslash(-1,1)^{n}$.

Proposition 6.2 implies that if $U \subset \mathbb{R}^{n}$ is an open definable set, then $U$ is a regular cell if and only if $\bar{U}$ is an $n$-ball and the frontier $\bar{U} \backslash U$ is an $(n-1)$-sphere.

Theorem 2.2. Every non-empty semi-monotone set $U \subset \mathbb{R}^{n}$ is a regular $n$-cell.

We are going to prove Theorem 2.2 by induction on the dimension $n$ of a regular cell. For $n=1$ the statement is obvious. Assume it is true for $n-1$.

Lemma 2.3. Let $U \subset \mathbb{R}^{n}$ be a semi-monotone set. Let

$$
U_{0}:=U \cap X_{j,=, c}, \quad U_{+}:=U \cap X_{j,>, c}, \quad \text { and } \quad U_{-}:=U \cap X_{j,<, c}
$$

for some $1 \leq j \leq n$ and $c \in \mathbb{R}$. Then $\bar{U}_{+} \cap \bar{U}_{-}=\bar{U}_{0}$.

Proof. Suppose a point $\mathbf{x}=\left(x_{1}, \ldots, x_{n}\right) \in X_{j,=, c} \backslash \bar{U}_{0}$ belongs to $\bar{U}_{+} \cap \bar{U}_{-}$. Then there is an $\varepsilon>0$ such that an open cube centered at $\mathbf{x}$,

$$
C_{\varepsilon}:=\bigcap_{1 \leq j \leq n}\left\{\left(y_{1}, \ldots, y_{n}\right)|| x_{j}-y_{j} \mid<\varepsilon\right\} \subset \mathbb{R}^{n},
$$

has non-empty intersections with both $U_{+}$and $U_{-}$and an empty intersection with $U_{0}$. Thus, $C_{\varepsilon} \cap U$ is not connected, which is not possible since, according to Corollary 1.4(1), $C_{\varepsilon} \cap U$ is semi-monotone. 
Corollary 2.4. Let $U \subset \mathbb{R}^{n}$ be a semi-monotone set. If $U_{+}$and $U_{-}$in Lemma 2.3 are regular cells, then $U$ is a regular cell.

Proof. We need to prove that $\bar{U}$ is a closed $n$-ball, and that the frontier $\bar{U} \backslash U$ is an $(n-1)$-sphere. The only non-trivial case is when $U_{0}$ is non-empty.

Since $U_{0}$ is semi-monotone due to Corollary $1.4, U_{0}$ is a regular $(n-1)$-cell by the inductive hypothesis. It follows that $\bar{U}_{0}, \bar{U}_{+}$, and $\bar{U}_{-}$are closed balls, while $\bar{U}_{0} \backslash U$ is an $(n-2)$-sphere. Hence $\bar{U}$ is obtained by gluing together two closed $n$-balls, $\bar{U}_{+}$and $\bar{U}_{-}$, along the closed $(n-1)$-ball $\bar{U}_{0}$ (see Definition 6.1). Proposition 6.4 implies that $\bar{U}$ is a closed $n$-ball.

According to Proposition 6.3, the sets $\bar{U}_{+} \backslash U=\partial \bar{U}_{+} \backslash U_{0}$ and $\bar{U}_{-} \backslash U=\partial \bar{U}_{-} \backslash U_{0}$ are closed $(n-1)$-balls. The frontier $\bar{U} \backslash U$ of $U$ is obtained by gluing $\bar{U}_{+} \backslash U$ and $\bar{U}_{-} \backslash U$ along the set $\left(\bar{U}_{+} \cap \bar{U}_{-}\right) \backslash U$, which, by Lemma 2.3 , is equal to $\bar{U}_{0} \backslash U$, and thus is an $(n-2)$-sphere, the common boundary of $\bar{U}_{+} \backslash U$ and $\bar{U}_{-} \backslash U$. It follows from Proposition 6.2 that $\bar{U} \backslash U$ is an $(n-1)$-sphere.

Lemma 2.5. If $U$ and $U_{-}$in Lemma 2.3 are regular cells, then $U_{+}$is also a regular cell.

Proof. Proposition 6.5 implies that $\bar{U}_{+}$is a closed $n$-ball. By the inductive hypothesis, $U_{0}$ is a regular cell. By Proposition $6.3, \bar{U}_{+} \backslash U=\partial \bar{U}_{+} \backslash U_{0}$ is a closed $(n-1)$-ball. Then the frontier $\bar{U}_{+} \backslash U_{+}$of $U_{+}$is obtained by gluing two closed $(n-1)$-balls, $\bar{U}_{+} \backslash U$ and $\bar{U}_{0}$, along the $(n-2)$-sphere $\bar{U}_{0} \backslash U$. Therefore, by Proposition 6.2, the frontier of $U_{+}$is an $(n-1)$-sphere.

Lemma 2.6. Let $n>5$ and $U \subset \mathbb{R}^{n}$ be a semi-monotone set and a regular cell. Then, for a generic c, both $U_{+}$and $U_{-}$in Lemma 2.3 are regular cells.

Proof. The set $U_{0}$ is a regular cell by the inductive hypothesis. Due to the theorem on triangulation of definable functions ([2, Th. 4.5]) applied to the projection of the $x_{j}$-coordinate function, there is a triangulation of $\bar{U}$ and a neighbourhood $(a, b)$ of $c$ in $\mathbb{R}$ such that the polyhedra corresponding to $\bar{U} \cap\left((a, b) \times \mathbb{R}^{n-1}\right)$ and $\bar{U}_{0} \times(a, b)$ are PL-homeomorphic. Hence, the $(n-1)$-sphere $\partial U_{0}$ is locally flatly embedded in the $n$ sphere $\partial U$. The lemma now follows from Proposition 6.10.

Let $\mathbb{R}_{+}^{n}$ be the open first octant $\left\{\left(x_{1}, \ldots, x_{n}\right) \in \mathbb{R}^{n} \mid x_{j}>0\right.$ for all $\left.1 \leq j \leq n\right\}$.

Lemma 2.7. Let $U$ be a semi-monotone set in $\mathbb{R}_{+}^{n}$ such that the origin is in $\bar{U}$. Let $c(t)=$ $\left(c_{1}(t), \ldots, c_{n}(t)\right)$ be a germ of a generic definable curve inside $U$ converging to the origin as $t \rightarrow 0$. Then

$$
U_{t}:=U \cap\left\{x_{1}<c_{1}(t), \ldots, x_{n}<c_{n}(t)\right\}
$$

is a regular cell for all small positive $t$.

Proof. By the inductive hypothesis of the induction on $n$, for every $1 \leq i \leq n$ each $(n-i)$-dimensional semi-monotone set

$$
C_{j_{1}, \ldots, j_{i}, t}:=U \cap\left\{x_{j_{1}}=c_{j_{1}}(t), \ldots, x_{j_{i}}=c_{j_{i}}(t), x_{k}<c_{k}(t) \text { for all } k \neq j_{1}, \ldots, j_{i}\right\},
$$


where $1 \leq j_{1}<\cdots<j_{i} \leq n$, is a regular $(n-i)$-cell. Since $c(t) \in U$, all sets $C_{j_{1}, \ldots, j_{i}, t}$ are non-empty.

Due to the theorem on triangulation of definable functions ([2, Th. 4.5]), for all small positive $t, \bar{U}_{t}$ is definably homeomorphic to a closed cone with vertex at the origin and with base definably homeomorphic to $\bar{D}_{t}$, where $D_{t}$ is the $(n-1)$-dimensional regular cell complex formed by the cells $C_{j_{1}, \ldots, j_{i}, t}$ for all $1 \leq j_{1}<\cdots<j_{i} \leq n$. Hence it is enough to prove that $D_{t}$ is shellable (see Definition 6.6), and therefore is a regular cell due to Proposition 6.7. We prove by induction on $k=1, \ldots, n$ a more general claim that the regular cell complex $D_{k, t}$ formed by the cells $C_{j_{1}, \ldots, j_{i}, t}, 1 \leq j_{1}<\cdots<j_{i} \leq k$, is shellable.

The base case $k=1$ holds because $C_{1, t}$ is a regular $(n-1)$-cell. By the inductive hypothesis on $n$, the set

$$
C_{i, j, t}=U \cap\left\{x_{i}=c_{i}(t), x_{j}=c_{j}(t), x_{k}<c_{k}(t) \text { for all } k \neq i, j\right\}
$$

is a regular $(n-2)$-cell. Since the germ $c(t)$ is generic, $\bar{C}_{i, j, t}=\bar{C}_{i, t} \cap \bar{C}_{j, t}$. Hence $D_{2, t}$ is obtained by gluing together two regular $(n-1)$-cells, $C_{1, t}$ and $C_{2, t}$, along the regular $(n-2)$-cell $C_{1,2, t}$ which is their common boundary (see Definition 6.1). It follows that the cell complex $D_{2, t}$ is shellable.

By the inductive hypothesis the complex $D_{k, t}$ is shellable. The set $C_{k+1, t}$ is a regular $(n-1)$-cell whose common boundary with $D_{k, t}$ is the $(n-2)$-dimensional shellable complex formed by $k$ regular $(n-2)$-cells $C_{1, k+1, t}, \ldots, C_{k, k+1, t}$. By Proposition 6.7, this common boundary is a regular $(n-2)$-cell. Hence, by Proposition 6.7 again, the complex $D_{k+1, t}$ is shellable.

Lemma 2.8. Let $U \subset \mathbb{R}_{+}^{n}$ be a semi-monotone set, with $n \leq 5$, such that the origin is in $\bar{U}$, and let $c(t)=\left(c_{1}(t), \ldots, c_{n}(t)\right)$ be a germ of a generic definable curve inside $\mathbb{R}_{+}^{n}$ (not necessarily inside $U$ ) converging to the origin as $t \rightarrow 0$. Then

$$
U_{t}=U \cap\left\{x_{1}<c_{1}(t), \ldots, x_{n}<c_{n}(t)\right\}
$$

is a regular cell for all small positive $t$.

Proof. We can repeat the proof of Lemma 2.7 if we prove that the regular cell complex $D_{t}$ formed by the non-empty sets

$$
C_{j_{1}, \ldots, j_{i}, t}:=U \cap\left\{x_{j_{1}}=c_{j_{1}}(t), \ldots, x_{j_{i}}=c_{j_{i}}(t), x_{k}<c_{k}(t) \text { for all } k \neq j_{1}, \ldots, j_{i}\right\},
$$

where $1 \leq j_{1}<\cdots<j_{i} \leq n$, is shellable. The difference from the proof of Lemma 2.7 is that here some of the sets $C_{j_{1}, \ldots, j_{i}, t}$ may be empty.

By Corollary 1.5 , the semi-monotone cell $U_{t}$ is acyclic. Hence so is $D_{t}$, for all small $t>0$.

Since $U$ is open, if $C_{j_{1}, \ldots, j_{k}, t}$ is non-empty then $C_{i_{1}, \ldots, i_{l}, t}$ is non-empty for any subset $\left\{i_{1}, \ldots, i_{l}\right\}$ of $\left\{j_{1}, \ldots, j_{k}\right\}$. It follows that the complex $D_{t}$ can be represented as a simplicial subcomplex $X$ of an $(n-1)$-simplex $\Delta$ so that every non-empty set $C_{j_{1}, \ldots, j_{i}, t}$ corresponds to the $(i-1)$-face of $\Delta$ having vertices $j_{1}, \ldots, j_{i}$. 
Observe that $X$ is acyclic since $D_{t}$ is acyclic. We prove by induction on the number of simplices in $X$ that the acyclicity of $X$ implies that $D_{t}$ is shellable. The base of the induction, for a single vertex, is trivial. According to Proposition $6.12, X$ has a vertex $v$ with an acyclic link $L$. The vertex $v$ corresponds to a regular $(n-1)$-cell $C_{j, t}$, while the link $L$ corresponds to the $(n-2)$-subcomplex of $D_{t}$ along which $C_{j, t}$ is glued to $D_{t}$. By the inductive hypothesis applied to $L$, that subcomplex of $D_{t}$ is shellable, and thus, by Proposition 6.7, is a regular cell. Removing the star of $v$ in $X$, we get a subcomplex $Y$ of $X$ which is acyclic by the Mayer-Vietoris exact sequence. By the inductive hypothesis, the subcomplex of $D_{t}$ corresponding to $Y$ is shellable. We have proved that $D_{t}$ is obtained by gluing a regular cell to a shellable complex along a regular cell, hence $D_{t}$ is shellable.

Lemma 2.9. Let $U \subset \mathbb{R}_{+}^{n}$ be a semi-monotone set, with $n \leq 5$, such that the origin is in $\bar{U}$, and let $c=\left(c_{1}, \ldots, c_{n}\right) \in \mathbb{R}_{+}^{n}$. Then $U_{c}:=U \cap\left\{x_{1}<c_{1}, \ldots, x_{n}<c_{n}\right\}$ is a regular cell for generic $c$ with $\|c\|$ small.

Proof. Consider a definable set $U_{\mathbf{y}}:=U \cap\left\{x_{1}<y_{1}, \ldots, x_{n}<y_{n}\right\} \subset \mathbb{R}_{+}^{2 n}$ with coordinates $x_{1}, \ldots, x_{n}, y_{1}, \ldots, y_{n}$ and $\mathbf{y}=\left(y_{1}, \ldots, y_{n}\right)$. By Corollary 6.15 , there is a partition of $\mathbb{R}_{+}^{n}$ (having coordinates $y_{1}, \ldots, y_{n}$ ) into definable sets $T$ such that if any $T$ is fixed, then for all $\mathbf{y} \in T$ the closures $\bar{U}_{\mathbf{y}}$ are definably homeomorphic to the same polyhedron, and the frontiers $\bar{U}_{\mathbf{y}} \backslash U_{\mathbf{y}}$ are definably homeomorphic to the same polyhedron.

For every $n$-dimensional $T$ such that the origin is in $\bar{T}$, there is, by the curve selection lemma ([2, Th. 3.2]), a germ of a generic definable curve $c(t)$ converging to 0 as $t \rightarrow 0$. Hence, by Lemma 2.8, for each $c \in T$ the set $\bar{U}_{c}$ is a closed $n$-ball, while $\bar{U}_{c} \backslash U_{c}$ is an $(n-1)$-sphere. Therefore, $U_{c}$ is a regular cell.

Lemma 2.10. Using the notation from Lemma 2.9, for $n \leq 5$, and for generic $c \in \mathbb{R}_{+}^{n}$ with $\|c\|$ small, the intersection

$$
U_{c} \cap \bigcap_{1 \leq \nu \leq k}\left\{x_{j_{v}} \sigma_{\nu} a_{v}\right\}
$$

for any $j_{v} \in\{1, \ldots, n\}, \sigma_{v} \in\{<,>\}$, and for any generic $a_{1} \geq \cdots \geq a_{k}$, is either empty or a regular cell.

Proof. It is sufficient to assume that $a_{v}<c_{j_{v}}$ for all $v$. We use induction on $k$. For $k=1$, the set $U_{c} \cap\left\{x_{j_{1}}<a_{1}\right\}$ is itself a set of the kind $U_{c}$, and therefore is a regular cell, by Lemma 2.9. Then the set $U_{c} \cap\left\{x_{j_{1}}>a_{1}\right\}$ is a regular cell due to Corollary 2.5.

By the inductive hypothesis, every non-empty set of the kind

$$
U_{c}^{(k-1)}:=U_{c} \cap \bigcap_{1 \leq \nu \leq k-1}\left\{x_{j_{v}} \sigma_{\nu} a_{\nu}\right\}
$$

is a regular cell. Also by the inductive hypothesis, replacing $c_{j_{k}}$ by $a_{k}$ if $a_{k}<c_{j_{k}}$, every set $U_{c}^{(k-1)} \cap\left\{x_{j_{k}}<a_{k}\right\}$ is a regular cell. Since both $U_{c}^{(k-1)}$ and $U_{c}^{(k-1)} \cap\left\{x_{j_{k}}<a_{k}\right\}$ are regular cells, so is $U_{c}^{(k-1)} \cap\left\{x_{j_{k}}>a_{k}\right\}$, by Corollary 2.5, which completes the induction.

In the similar statement for $n>5$ we need to assume that $c$ is a generic point in $U$. 
Lemma 2.11. Let $U$ be a semi-monotone set in $\mathbb{R}_{+}^{n}$, with $n>5$, let $c=\left(c_{1}, \ldots, c_{n}\right)$ be a generic point in $U$, and let $U_{c}:=U \cap\left\{x_{1}<c_{1}, \ldots, x_{n}<c_{n}\right\}$. Then

$$
U_{c} \cap \bigcap_{1 \leq \nu \leq k}\left\{x_{j_{v}} \sigma_{\nu} a_{\nu}\right\}
$$

for $\|c\|$ small, for any $j_{v} \in\{1, \ldots, n\}, \sigma_{v} \in\{<,>\}$, and for any generic $a_{1} \geq \cdots \geq a_{k}$, is either empty or a regular cell.

Proof. Lemma 2.7 and the argument from the proof of Lemma 2.9 imply that $U_{c}$ is a regular cell. We argue by induction on $k$. For $k=1$, both sets $U_{c} \cap\left\{x_{j_{1}}>a_{1}\right\}$ and $U_{c} \cap\left\{x_{j_{1}}<a_{1}\right\}$ are regular cells, due to Lemma 2.6. Assume by the inductive hypothesis that every non-empty set of the kind $U_{c}^{(k-1)}$ (see (2.1)) is a regular cell. Then, due to Lemma 2.6, both $U_{c}^{(k-1)} \cap\left\{x_{j_{k}}>a_{k}\right\}$ and $U_{c}^{(k-1)} \cap\left\{x_{j_{k}}<a_{k}\right\}$ are regular cells.

Lemma 2.12. Let $U \subset \mathbb{R}^{n}$ be a semi-monotone set, and let $\mathbf{y}=\left(y_{1}, \ldots, y_{n}\right) \in \bar{U}$. Then for generic points $a=\left(a_{1}, \ldots, a_{n}\right), b=\left(b_{1}, \ldots, b_{n}\right) \in \mathbb{R}_{+}^{n}$ with $\|a\|$ and $\|b\|$ small, the intersection

$$
U_{a, b}:=U \cap \bigcap_{1 \leq j \leq n}\left\{-a_{j}<x_{j}-y_{j}<b_{j}\right\}
$$

is a regular cell.

Proof. We use induction on $n$, with the case $n=1$ being obvious.

Translate the point $\mathbf{y}$ to the origin. Let $c=\left(c_{1}, \ldots, c_{m}\right) \in \mathbb{R}^{n}$ be a generic point, and $\mathbb{P}_{n, c}$ the open octant of $\mathbb{R}^{n}$ containing $c$. By Lemma 2.9 in the case $n \leq 5$, or by Lemma 2.11 in the case $n>5$, for a generic point $c=\left(c_{1}, \ldots, c_{n}\right) \in \mathbb{P}_{n, c} \cap U$ with $\|c\|$ small, the set $U_{c}:=U \cap \mathbb{P}_{n, c} \cap\left\{\left|x_{1}\right|<\left|c_{1}\right|, \ldots,\left|x_{n}\right|<\left|c_{n}\right|\right\}$ is either empty or a regular cell. Choose such a point $c$ in every octant of $\mathbb{R}^{n}$.

Choose $-a_{i}$ (respectively, $b_{i}$ ) to be the maximum (respectively, minimum) among the negative (respectively, positive) $c_{i}$ over all octants $\mathbb{P}$. We now prove that, with $a$ and $b$ so chosen, the set $U_{a, b}$ is a regular cell. We use induction on $r=0, \ldots, n-1$. For $r=0$, if $d$ is a vertex of

$$
\bigcap_{1 \leq j \leq n}\left\{-a_{j}<x_{j}<b_{j}\right\}
$$

belonging to one of the $2^{n}=2^{n-r}$ octants $\mathbb{P}$, then $U_{d}$ is either empty or a regular cell (by Lemma 2.10 for $n \leq 5$, and Lemma 2.11 for $n>5$ ). Partition the family of all sets of the kind $U_{d}$ into pairs $\left(U_{d^{\prime}}, U_{d^{\prime \prime}}\right)$ so that $d_{1}^{\prime}=a_{1}, d_{1}^{\prime \prime}=b_{1}$ and $d_{i}^{\prime}=d_{i}^{\prime \prime}$ for all $i=2, \ldots, n$. Whenever the cells $U_{d^{\prime}}, U_{d^{\prime \prime}}$ are both non-empty, they have a common $(n-1)$-face

$$
U \cap\left(\{0\} \times \mathbb{P}_{n-1,\left(d_{2}^{\prime}, \ldots, d_{n}^{\prime}\right)}\right) \cap\left\{x_{1}=0,\left|x_{2}\right|<d_{2}^{\prime}, \ldots,\left|x_{n}\right|<d_{n}^{\prime}\right\},
$$

which, by the inductive hypothesis of the induction on $n$, is a regular cell. Then, according to Corollary 2.4, the union of the common face and $U_{d^{\prime}} \cup U_{d^{\prime \prime}}$ is a regular cell. Gluing in this way all pairs $\left(U_{d^{\prime}}, U_{d^{\prime \prime}}\right)$, we get a family of $2^{n-1}$ sets which are either empty or regular cells. This family is partitioned into pairs of regular cells each of which has a common regular cell face in the hyperplane $\left\{x_{2}=0\right\}$. In the last step of the induction, for 
$r=n-1$, we are left with at most two regular cells having, in the case of exactly two cells, a common regular cell face in the hyperplane $\left\{x_{n}=0\right\}$. Gluing these sets along the common face, we get, by Corollary 2.4 , the regular cell $U_{a, b}$.

Lemma 2.13. Using the notations from Lemma 2.12, the intersection

$$
V_{a, b}:=U_{a, b} \cap \bigcap_{1 \leq \nu \leq k}\left\{x_{j_{v}} \sigma_{\nu} d_{\nu}\right\}
$$

for any $j_{v} \in\{1, \ldots, n\}, \sigma_{v} \in\{<,>\}$, and for any generic $d_{1} \geq \cdots \geq d_{k}$, is either empty or a regular cell.

Proof. Analogous to the proof of Lemmas 2.10 for $n \leq 5$, and to the proof of Lemma 2.11 for $n>5$.

Proof of Theorem 2.2. For each point $\mathbf{y} \in \bar{U}$ choose generic points $a, b \in \mathbb{R}^{n}$ as in Lemma 2.12, so that the set $U_{a, b}$ becomes a regular cell. We get an open covering of the compact set $\bar{U}$ by sets of the kind

$$
A_{a, b}=: \bigcap_{1 \leq j \leq n}\left\{-a_{j}<x_{j}-y_{j}<b_{j}\right\} ;
$$

choose any finite subcovering $\mathcal{C}$. For every $j=1, \ldots, n$ consider the finite set $D_{j}$ of $j$-coordinates $a_{j}, b_{j}$ for all sets $A_{a, b}$ in $\mathcal{C}$. Let

$$
\bigcup_{1 \leq j \leq n} D_{j}=\left\{d_{1}, \ldots, d_{k}\right\}
$$

with $d_{1} \geq \cdots \geq d_{k}$. Every set $V_{a, b}$, corresponding to $\left\{d_{1}, \ldots, d_{k}\right\}$ (see (2.2)), is regular, by Lemma 2.13, and $U$ is the union of those $V_{a, b}$ and their common faces for which $A_{a, b} \in \mathcal{C}$.

The rest of the proof is similar to the final part of the proof of Lemma 2.12. Use induction on $r=1, \ldots, n$, within the current induction step of the induction on $n$. The base of the induction is for $r=1$. Let $D_{1}=\left\{d_{1,1}, \ldots, d_{1, k_{1}}\right\}$ with $d_{1,1} \geq \cdots \geq d_{1, k_{1}}$. Partition the finite family of all regular cells $V_{a, b}$, for all $A_{a, b} \in \mathcal{C}$, into $\left(\left|D_{1}\right|-1\right)$-tuples so that the projections of cells in a tuple on the $x_{1}$-coordinate are exactly the intervals

$$
\left(d_{1, k_{1}}, d_{1, k_{1}-1}\right),\left(d_{1, k_{1}-1}, d_{1, k_{1}-2}\right), \ldots,\left(d_{1,2}, d_{1,1}\right),
$$

and any two cells in a tuple having as projections two consecutive intervals in (2.3) have a common $(n-1)$-dimensional face in a hyperplane $\left\{x_{1}=\right.$ const $\}$. This face, by the external inductive hypothesis (of the induction on $n$ ), is a regular cell. According to Corollary 2.4, the union of any two consecutive cells and their common face is a regular cell. Gluing in this way all consecutive pairs in every $\left(\left|D_{1}\right|-1\right)$-tuple, we get a smaller family of regular cells. This family, in the next induction step $r=2$, is partitioned into $\left(\left|D_{2}\right|-1\right)$-tuples of cells such that in each of these tuples two consecutive cells have a common regular cell face in a hyperplane $\left\{x_{2}=\right.$ const $\}$. In the last step, $r=n$, of the induction we are left with one $\left(\left|D_{n}\right|-1\right)$-tuple of regular cells such that two consecutive cells have a common regular cell face in a hyperplane $\left\{x_{n}=\right.$ const $\}$. Gluing all pairs of consecutive cells along their common faces, we get, by Corollary 2.4 , the regular cell $U$. 


\section{Semi-algebraic semi-monotone sets over real closed fields}

In this section we prove the regularity of semi-monotone sets for semi-algebraic sets defined over an arbitrary real closed field $\mathrm{R}$ which is fixed for the rest of the section. Accordingly, in the definition of semi-monotonicity, "connectivity" refers to "semi-algebraic connectivity", while an $n$-dimensional semi-algebraic regular cell $S \subset \mathrm{R}^{n}$ is such that there exists a semi-algebraic homeomorphism

$$
h:(\bar{S}, S) \rightarrow\left([-1,1]^{n},(-1,1)^{n}\right)
$$

(cf. Definition 2.1).

Definition 3.1. Let $S \subset \mathrm{R}^{n}$ be a semi-algebraic set. We say that the complexity of $S$ is bounded by a natural number $N$ if there exists a quantifier-free first-order formula $\Phi$ defining $S$ such that $N \geq s d$, where $s$ (respectively, $d$ ) is the the number (respectively, maximum degree) of the polynomials appearing in $\Phi$. By the complexity of a semi-algebraic map we mean the complexity of its graph.

The idea of the proof is to show that for a fixed $N$ the statement that "any semimonotone set with complexity $N$ is a regular cell" can be expressed by a first-order formula of the theory of R (with integer coefficients), and therefore is true as long as it is true for $\mathrm{R}=\mathbb{R}$, due to the Tarski-Seidenberg transfer principle ([1, Proposition 5.2.3]). (Note that the direct repetition for arbitrary $\mathrm{R}$ of the proof from Section 2 is probably impossible because $\mathrm{R}$ may be non-archimedean.)

Lemma 3.2. For any pair $\left(T_{1}, T_{2}\right)$ of semi-algebraic sets with $T_{2} \subset T_{1} \subset \mathrm{R}^{n}$, there exists a natural-valued function $F(N, n)$ with the following property. Let pairs $\left(S_{1}, S_{2}\right)$ and $\left(T_{1}, T_{2}\right)$ of semi-algebraic sets be semi-algebraically homeomorphic, where the sets $S_{2} \subset S_{1} \subset \mathrm{R}^{n}$ have complexities bounded by $N$, and $S_{1}$ is closed and bounded. Then there exists a semi-algebraic homeomorphism

$$
f:\left(S_{1}, S_{2}\right) \rightarrow\left(T_{1}, T_{2}\right)
$$

with complexity bounded by $F(N, n)$.

Proof. It follows from the theorem on triangulations of semi-algebraic sets ([1, Theorem 9.2.1]) that there exists a natural-valued function $H(N, n)$ having the following property. There exist a finite simplicial complex $K_{1}$ having at most $H(N, n)$ simplices, a union $K_{2}$ of its simplices, and a semi-algebraic homeomorphism

$$
h:\left(S_{1}, S_{2}\right) \rightarrow\left(\left|K_{1}\right|,\left|K_{2}\right|\right),
$$

such that the complexity of $h$ is also bounded by $H(N, n)$. Since the number of simplicial complexes having at most $H(N, n)$ simplices is finite, there is an $\mathbb{N}$-valued function $G(N, n)$ bounding the complexity of any semi-algebraic homeomorphism

$$
g:\left(\left|K_{1}\right|,\left|K_{2}\right|\right) \rightarrow\left(T_{1}, T_{2}\right)
$$


Thus, there exists a semi-algebraic homeomorphism

$$
f:=g \circ h:\left(S_{1}, S_{2}\right) \rightarrow\left(T_{1}, T_{2}\right)
$$

with complexity bounded by some $\mathbb{N}$-valued function $\phi$ of $H(N, n)$ and $G(N, n)$ which can be explicitly described using bounds on effective quantifier elimination. Define $F(N, n):=\phi(H(N, n), G(N, n))$.

One can consider a semi-algebraic subset in $\mathrm{R}^{m} \times \mathrm{R}^{n}$ as a semi-algebraic family of subsets of $\mathrm{R}^{n}$ parameterized by points of $\mathrm{R}^{m}$. Using again the theorem on triangulations of semialgebraic sets, it is easy to check that the family of all those semi-algebraic subsets of $\mathrm{R}^{n}$ of complexity bounded by $N$ which are semi-monotone is a semi-algebraic family.

Theorem 3.3. Let $\mathrm{R}$ be a real closed field. Every non-empty semi-algebraic semi-monotone subset of $\mathrm{R}^{n}$ is a semi-algebraic regular cell.

Proof. It suffices to prove the theorem for all non-empty semi-algebraic semi-monotone sets of complexity bounded by $N$ for each $N>0$. Fix $N$. Since the family of all such sets is a semi-algebraic family, Lemma 3.2 for

$$
\left(T_{1}, T_{2}\right)=\left([-1,1]^{n},(-1,1)^{n}\right)
$$

implies that the existence of the required homeomorphism is expressible as a sentence in the language of the first-order theory of the field $\mathrm{R}$ with integer coefficients. The TarskiSeidenberg transfer principle now implies that it suffices to prove the truth of this sentence for any one particular real closed field. The theorem follows since we have proved this sentence for $\mathrm{R}=\mathbb{R}$ in Theorem 2.2 .

\section{Regular cells in the sense of van den Dries are not regular}

In o-minimality theory the following classes of topological cells and continuous functions are considered, which are also based on the idea of monotonicity. In [7] these cells and functions are called regular, we will call them $v d D$-regular.

Definition 4.1 (cf. Theorem 1.7). An (open) cylindrical cell $X \subset \mathbb{R}^{n}$ is an open subset defined by induction as follows. For $n=0, X$ is the point. Let $X$ be a cylindrical cell in $\mathbb{R}^{n-1}$, and $f, g: X \rightarrow \mathbb{R}$ be two continuous functions such that $f(\mathbf{x})<g(\mathbf{x})$ for all $\mathbf{x} \in X$. Then $\{(\mathbf{x}, t) \mid \mathbf{x} \in Y, f(\mathbf{x})<t<g(\mathbf{x})\}$ is a cylindrical cell in $\mathbb{R}^{n+1}$.

Definition 4.2 ([7]). A cylindrical cell $X \subset \mathbb{R}^{n}$ is $v d D$-regular if for each $1 \leq i \leq n$, any two points $\mathbf{x}=\left(x_{1}, \ldots, x_{n}\right), \mathbf{y}=\left(y_{1}, \ldots, y_{n}\right) \in X$ and each point $\mathbf{z}=\left(z_{1}, \ldots, z_{n}\right) \in \mathbb{R}^{n}$ such that $x_{j}=y_{j}=z_{j}$ for all $j \neq i$, the condition $x_{i}<z_{i}<y_{i}$ implies $\mathbf{z} \in X$.

Let $X$ be a vdD-regular cell. A continuous function $f: X \rightarrow \mathbb{R}$ is $v d D$-regular if for each $1 \leq i \leq n$ it is either strictly increasing, strictly decreasing, or constant along the coordinate $i$. Here, $f$ is strictly increasing along the coordinate $i$ if for any two points $\mathbf{x}=\left(x_{1}, \ldots, x_{n}\right), \mathbf{y}=\left(y_{1}, \ldots, y_{n}\right) \in X$ such that $x_{j}=y_{j}$ for all $j \neq i$, and $x_{i}<y_{i}$, we have $f(\mathbf{x})<f(\mathbf{y})$. Similarly, we define functions strictly decreasing and functions constant along the coordinate $i$. 
The following example shows that a vdD-regular cell may not be regular, and that a vdD-regular function defined on a vdD-regular cell may not be supermonotone (or submonotone).

Example 4.3. Consider the 3-simplex

$$
X:=\left\{(x, y, z) \in \mathbb{R}^{3} \mid 0<x, 0<y, 0<z<1, x+y<z\right\},
$$

and the continuous function $h: X \rightarrow \mathbb{R}$ defined by

$$
h(x, y, z)=(x / z)^{2}+(y / z)^{2} .
$$

Observe that $X$ is a vdD-regular cylindrical cell, while $h$ is a vdD-regular function. It is easy to see that for every $t \in(1 / 2,1)$ the level set

$$
\{(x, y, z) \in X \mid h(x, y, z)=t\}
$$

is not connected, while its closure is a cone with vertex at the origin and base consisting of two disjoint arcs of a circle. Hence the graph of $h$ itself is not a regular cell. It follows that the vdD-regular cell

$$
\{(x, y, z, t) \mid(x, y, x) \in X, 0<t<h(x, y, z)\}
$$

is not a regular cell.

Note that the set $\{(x, y, z) \in X \mid h(x, y, z)>1 / 2\}$ consists of two connected components, and therefore is not semi-monotone. Therefore the vdD-regular function $h$ is not supermonotone.

\section{Semi-monotone sets and regular Boolean functions}

Consider a Boolean function $\psi=\psi\left(\xi_{1}, \ldots, \xi_{n}\right)$ in $n$ Boolean variables $\xi_{j} \in\{0,1\}$. For any $j=1, \ldots, n$ and $c \in\{0,1\}$, let $\psi_{j, c}$ be the restriction of $\psi$ to the subspace $\mathcal{C}_{j, c}=\left\{\xi_{j}=c\right\} \subset\{0,1\}^{n}$. The operations

$$
E_{j}(\psi)=\psi_{j, 0} \vee \psi_{j, 1} \quad \text { and } \quad A_{j}(\psi)=\psi_{j, 0} \wedge \psi_{j, 1}
$$

assign to $\psi$ two Boolean functions in $n-1$ variables. These operations can also be defined by the formulae $\exists \xi_{j} \psi\left(\xi_{1}, \ldots, \xi_{n}\right)$ and $\forall \xi_{j} \psi\left(\xi_{1}, \ldots, \xi_{n}\right)$, respectively.

Definition 5.1. A regular Boolean function is defined inductively as follows. Any univariate Boolean function is regular. A Boolean function $\psi\left(\xi_{1}, \xi_{2}\right)$ is regular if the set $\{\psi=1\}$ is neither $\{(1,0),(0,1)\}$ nor $\{(0,0),(1,1)\}$. Equivalently, $\psi\left(\xi_{1}, \xi_{2}\right)$ is regular if $E_{1}\left(A_{2}(\psi)\right)=A_{2}\left(E_{1}(\psi)\right)$ or $A_{1}\left(E_{2}(\psi)\right)=E_{2}\left(A_{1}(\psi)\right)$. For $n>2$, a Boolean function $\psi$ on $\{0,1\}^{n}$ is regular if the following two conditions are satisfied:

(R1) Restriction of $\psi$ to each Boolean square

$$
\left\{\xi_{j_{1}}=c_{1}, \ldots, \xi_{j_{n-2}}=c_{n-2}\right\}, \quad 1 \leq j_{1}<\cdots<j_{n-2} \leq n,
$$

is regular.

(R2) The functions $E_{n}(\psi)$ and $A_{n}(\psi)$ are regular. 
Lemma 5.2. Let $\psi\left(\xi_{1}, \xi_{2}, \xi_{3}\right)$ be a Boolean function such that the functions $\psi_{j, c}$ are regular for all $j=1,2,3$ and $c \in\{0,1\}$.

1. If $E_{3}(\psi)$ is not regular then $\{\psi=1\}$ is one of the four sets each consisting of two diagonally opposite vertices of $\{0,1\}$.

2. If $A_{3}(\psi)$ is not regular then $\{\psi=0\}$ is one of the four sets each consisting of two diagonally opposite vertices of $\{0,1\}^{3}$.

Proof. Straightforward checking.

Theorem 5.3. A Boolean function $\psi\left(\xi_{1}, \ldots, \xi_{n}\right)$ is regular if and only if the result of any sequence of operations $E_{j}$ and $A_{k}$ applied to $\psi$ does not depend on the order of the operations.

Proof. We prove the statement by induction on $n$. The case $n \leq 2$ follows immediately from the definition of a regular function. Let $n \geq 3$.

Suppose that for a function $\psi$ the result of any sequence of operations $E_{j}$ and $A_{k}$ applied to $\psi$ does not depend on the order of the operations. This immediately implies (R1). Since $E_{n}(\psi)$ and $A_{n}(\psi)$ are functions in $n-1$ variables, they are regular by the inductive hypothesis, i.e., condition (R2) is also true. Hence, $\psi$ is regular.

Conversely, let $\psi$ be a regular function. For any Boolean function $\chi$ and any $j \neq k$ we have $E_{j}\left(E_{k}(\chi)\right)=E_{k}\left(E_{j}(\chi)\right)$ and $A_{j}\left(A_{k}(\chi)\right)=A_{k}\left(A_{j}(\chi)\right)$. Condition (R1) implies that for regular $\psi$ and any $j \neq k$, the equality $E_{j}\left(A_{k}(\psi)\right)=A_{k}\left(E_{j}(\psi)\right)$ is true. Hence we only have to show that the functions $E_{j}(\psi)$ and $A_{j}(\psi)$ are regular for each $j<n$. We will only prove that $\varphi:=E_{j}(\psi)$ is regular. The proof for $A_{j}(\psi)$ is similar.

For $j<n$, the functions $\varphi_{n, 0}:=E_{j}\left(\psi_{n, 0}\right)$ and $\varphi_{n, 1}:=E_{j}\left(\psi_{n, 1}\right)$ are regular due to the induction hypothesis.

Since $E_{n}(\psi)$ is regular and $E_{n}(\varphi)=E_{j}\left(E_{n}(\psi)\right)$, the function $E_{n}(\varphi)$ is regular due to the induction hypothesis. Since $A_{n}(\psi)$ is regular and $A_{n}(\varphi)=E_{j}\left(A_{n}(\psi)\right)$ by condition (R1), the function $A_{n}(\varphi)$ is regular due to the induction hypothesis. Hence it remains to show that the restriction of $\varphi$ to any Boolean square $B$ in $\{0,1\}^{n-1}$ is regular. If $B$ has the value of $\xi_{n}$ fixed, this follows from the regularity of $\varphi_{n, 0}$ and $\varphi_{n, 1}$.

Suppose that the values of all variables except $\xi_{n}$ and $\xi_{k}$, for some $j \neq k<n-1$, are fixed on $B$, and the restriction of $\varphi$ to $B$ is not regular. Then the intersection of $\{\psi=1\}$ with the corresponding Boolean 3 -cube $C$ in $\{0,1\}^{n}$ (with the values of all variables except $\xi_{n}, \xi_{k}$ and $\xi_{j}$ fixed) consists of two diagonally opposite vertices due to Lemma 5.2. Hence the restriction of $E_{n}(\psi)$ to the projection of $C$ along $\xi_{n}$ is not regular, which contradicts regularity of $E_{n}(\psi)$.

Corollary 5.4. Any regular Boolean function $\psi$ remains regular under any permutation of the variables, replacing any $\xi_{j}$ by $1-\xi_{j}$, replacing $\xi_{j}$ by a constant $c \in\{0,1\}$ for any $j \in\{1, \ldots, n\}$, and replacing $\psi$ by $1-\psi$.

Proof. Straightforward.

Consider the cube $[-1,1]^{n} \subset \mathbb{R}^{n}$ as a union of $2^{n}$ closed unit cubes with the common vertex at the origin. Shifting the center of a unit cube by $(1 / 2, \ldots, 1 / 2)$ assigns a point in $\{0,1\}^{n}$ to this unit cube. In this way, the unit cubes correspond bijectively to the points of $\{0,1\}^{n}$. 
Theorem 5.5. A Boolean function $\psi\left(\xi_{1}, \ldots, \xi_{n}\right) \not \equiv 0$ is regular if and only if the union $C_{\psi}$ of closed unit cubes corresponding to points $\left(\xi_{1}, \ldots, \xi_{n}\right) \in\{\psi=1\}$ is a closed PL n-ball.

Proof. We use induction on $n$, where the case $n=2$ follows from Definition 5.1.

Suppose a Boolean function $\psi$ is regular.

Let $C_{\psi+}$ (respectively, $C_{\psi-}$ ) be the union of all unit cubes corresponding to vertices with $\xi_{n}=1$ (respectively, $\xi_{n}=0$ ). Since, due to Corollary 5.4, the functions $\psi\left(\xi_{1}, \ldots, \xi_{n-1}, 1\right)$ and $\psi\left(\xi_{1}, \ldots, \xi_{n-1}, 0\right)$ are regular, both $C_{\psi+}$ and $C_{\psi-}$ are closed PL $n$-balls, by the inductive hypothesis. Due to Proposition 6.4, it is sufficient to prove that the intersection

$$
C_{\psi 0}:=C_{\psi+} \cap C_{\psi-} \subset[-1,1]^{n} \cap\left\{x_{n}=0\right\}
$$

is a closed PL $(n-1)$-ball.

If $C_{\psi 0}$ is pure $(n-1)$-dimensional, then $C_{\psi 0}=C_{A_{n}(\psi)}$. Since $A_{n}(\psi)$ is regular, $C_{\psi 0}$ is a PL $(n-1)$-ball, by the inductive hypothesis.

We now show that $C_{\psi 0}$ is indeed pure $(n-1)$-dimensional. Suppose that, on the contrary, $C_{\psi 0}$ contains a common $m$-face $F$ of a unit cube in $C_{\psi+}$ and a unit cube in $C_{\psi-}$, with $0 \leq m<n$, and $F$ is not contained in any common face of a larger dimension.

Let $m>0$. Then for some $1 \leq i \leq n-1$ and $c \in\{0,1\}$ the set $F \cap\left\{x_{i}=c\right\}$ is a common (m-1)-face of some unit cubes in $C_{\psi+} \cap\left\{x_{i}=c\right\}$ and $C_{\psi+} \cap\left\{x_{i}=c\right\}$, which is not contained in any common face of a larger dimension. Hence, for the restriction $\psi_{i, c}$ of $\psi$ on $\left\{\xi_{i}=c\right\}$, the set $C_{\psi_{i, c}}$ is not a PL $(n-1)$-cube, therefore, by the inductive hypothesis, $\psi_{i, c}$ is not regular. This contradicts Corollary 5.4.

Now, let $m=0$. This can only happen when each of $C_{\psi+}$ and $C_{\psi-}$ consists of just one cube, and this pair of cubes corresponds to diagonally opposite vertices of $[-1,1]^{n}$. Then $\psi$ is not regular, which is a contradiction.

Conversely, suppose for a Boolean function $\psi$ the set $C_{\psi}$ is a PL ball. Then for all $1 \leq i \leq n$ and $c \in\{0,1\}$ the sets $C_{\psi_{i, c}}$ are also PL $(n-1)$-balls, hence, by the inductive hypothesis, all functions $\psi_{i, c}$ are regular. This implies condition (R1) for $\psi$.

The set $C_{E_{n}(\psi)}$ is the projection of $C_{\psi}$ along the coordinate $x_{n}$, and is therefore a PL $(n-1)$-ball. Hence $E_{n}(\psi)$ is a regular function by the inductive hypothesis. The intersection of the two PL $n$-balls $C_{\psi+}$ and $C_{\psi-}$, defined above, is a PL $(n-1)$-ball, and it coincides with $C_{A_{n}(\psi)}$. Therefore $A_{n}(\psi)$ is a regular function by the inductive hypothesis. Thus condition (R2) is also satisfied, and $\psi$ is regular by definition.

Corollary 5.6. For a regular Boolean function $\psi$,

(1) $A_{j}(\psi) \equiv 0$ if and only if either $\psi_{j, 0} \equiv 0$ or $\psi_{j, 1} \equiv 0$;

(2) $E_{j}(\psi) \equiv 1$ if and only if either $\psi_{j, 0} \equiv 1$ or $\psi_{j, 1} \equiv 1$.

Proof. (1) Let $C_{\psi, j+}$ (respectively, $C_{\psi, j-}$ ) be the union of all unit cubes corresponding to vertices with $\xi_{j}=1$ (respectively, $\xi_{j}=0$ ). Since $A_{j}(\psi)=\psi_{j, 0} \wedge \psi_{j, 1} \equiv 0$, the set $C_{\psi}$ cannot contain two unit cubes corresponding to vertices differing only in the $j$ th coordinate. It follows that if both $C_{\psi, j+}$ and $C_{\psi, j-}$ are non-empty, then $\operatorname{dim}\left(C_{\psi, j+} \cap C_{\psi, j-}\right)<$ $n-1$. This contradicts the fact that $C_{\psi}$ is a PL $n$-ball. 
The converse statement is trivial.

(2) follows from (1) and De Morgan's law: $E_{j}(\psi) \equiv 1-A_{j}(-\psi)$.

Definition 5.7. Let $p \in \mathbb{R}^{n}$. The finite set $\mathcal{Z}$ of octants with vertex at $p$ corresponds to a Boolean function $\psi$ if, when translated to 0 , the octants in $\mathcal{Z}$ contain exactly all unit cubes in $[-1,1]^{n}$ corresponding to points $\left(\xi_{1}, \ldots, \xi_{n}\right) \in\{\psi=1\}$.

Let $U \subset \mathbb{R}^{n}$. If the set $\mathcal{Z}$ of all octants with the vertex at $p$ and having non-empty intersections with $U$ corresponds to a Boolean function $\psi$, then we say that $\psi$ is the Boolean function for $U$ at $p$.

Lemma 5.8. Let $U \subset \mathbb{R}^{n}$ be a non-empty semi-monotone set, and $U^{\prime}$ be its projection along the coordinate $x_{n}$. If $p^{\prime} \notin U^{\prime}$ and the Boolean function $\varphi$ for $U^{\prime}$ at $p^{\prime}$ is $\varphi \equiv 1$, then there exists $p_{n} \in \mathbb{R}$ such that the Boolean function $\psi$ for $U$ at $p=\left(p^{\prime}, p_{n}\right)$ is either not regular or $\psi \equiv 1$.

Proof. Let $p^{\prime} \notin U^{\prime}$ and $\varphi \equiv 1$. Suppose that for every $p_{n} \in \mathbb{R}$ the function $\psi$ is regular. Since $\varphi=E_{n}(\psi)$ for any $p_{n} \in \mathbb{R}$, Corollary 5.6 implies that for every $p_{n}$ either $\psi_{j, 0} \equiv 1$, or $\psi_{j, 1} \equiv 1$. Observe that $\psi_{j, 0} \equiv 1$ for all sufficiently large values of $p_{n}$, while $\psi_{j, 1} \equiv 1$ for all sufficiently small values of $p_{n}$. Therefore there exists an intermediate value of $p_{n}$ for which $\psi \equiv 1$.

Theorem 5.9. A non-empty open set $U \subset \mathbb{R}^{n}$ is semi-monotone if and only if for every point $p=\left(p_{1}, \ldots, p_{n}\right) \in \mathbb{R}^{n} \backslash U$ the Boolean function $\psi$ for $U$ at $p$ is a non-constant regular function.

Proof. Suppose that $U$ is semi-monotone and $p=\left(p_{1}, \ldots, p_{n}\right) \in \mathbb{R}^{n} \backslash U$. Let $\psi$ be the Boolean function for $U$ at $p$, and let $\mathcal{Z}$ correspond to $\psi$.

According to Theorem 5.5, it is sufficient to prove that the union $C$ of all unit cubes in $[-1,1]^{n}$ corresponding to octants from $\mathcal{Z}$ is a closed PL $n$-ball different from the whole $[-1,1]^{n}$. We prove this by induction on $n$, with the case $n=1$ being trivial.

Let $C=C_{+} \cup C_{-}$where $C_{+}$(respectively, $C_{-}$) is the union of all unit cubes corresponding to vertices of $[-1,1]^{n}$ with $\xi_{n}=1$ (respectively, $\xi_{n}=0$ ).

The projection $U_{+}^{\prime}$ (respectively, $U_{-}^{\prime}$ ) of $U \cap X_{n,>, p_{n}}$ (respectively, of $U \cap X_{n,<, p_{n}}$ ) along the coordinate $x_{n}$ is semi-monotone due to Proposition 1.2. If $\left(p_{1}, \ldots, p_{n-1}\right) \in U_{ \pm}^{\prime}$ then the projection of $C_{ \pm}$along $x_{n}$ coincides with $[-1,1]^{n-1}$. Otherwise, by the inductive hypothesis, the projection of $C_{ \pm}$along $x_{n}$ is a closed PL $(n-1)$-ball. In any case, the set $C_{ \pm}$itself is a closed PL $n$-ball.

By Proposition 6.4, it is sufficient to prove that the intersection $C_{0}:=C_{+} \cap C_{-}$is a closed PL $(n-1)$-ball. The same argument as in the proof of Theorem 5.5 shows that $C_{0}$ is pure $(n-1)$-dimensional. We now prove that the set $C_{0}$ coincides with the union of the unit $(n-1)$-cubes for $U \cap X_{n,=, p_{n}}$. Indeed, if for two octants

$$
D_{+}:=X_{1, \sigma_{1}, p_{1}} \cap \cdots \cap X_{n-1, \sigma_{n-1}, p_{n-1}} \cap X_{n,>, p_{n}}
$$

and

$$
D_{-}:=X_{1, \sigma_{1}, p_{1}} \cap \cdots \cap X_{n-1, \sigma_{n-1}, p_{n-1}} \cap X_{n,<, p_{n}},
$$


where $\sigma_{1}, \ldots, \sigma_{n-1} \in\{<,>\}$, both intersections $D_{+} \cap U$ and $D_{-} \cap U$ are non-empty, then $U \cap X_{n,=, p_{n}}$ is also non-empty since

$$
U \cap X_{1, \sigma_{1}, p_{1}} \cap \cdots \cap X_{n-1, \sigma_{n-1}, p_{n-1}}
$$

is semi-monotone and therefore connected. By the inductive hypothesis, $C_{0}$ is a closed PL $(n-1)$-ball.

It remains to show that $C \neq[-1,1]^{n}$. By the inductive hypothesis, $C_{0} \neq[-1,1]^{n-1}$. It follows that

$$
U \cap X_{1, \sigma_{1}, p_{1}} \cap \cdots \cap X_{n-1, \sigma_{n-1}, p_{n-1}} \cap X_{n,=, p_{n}}=\emptyset
$$

for some $\sigma_{1}, \ldots, \sigma_{n-1} \in\{<,>\}$. If $C=[-1,1]^{n}$, then $U \cap D_{+} \neq \emptyset$ and $U \cap D_{-} \neq \emptyset$. Hence the semi-monotone set

$$
U \cap X_{1, \sigma_{1}, p_{1}} \cap \cdots \cap X_{n-1, \sigma_{n-1}, p_{n-1}}
$$

is not connected, which is a contradiction.

Conversely, suppose that for every $p \in \mathbb{R}^{n} \backslash U$ the Boolean function $\psi$ for $U$ at $p$ is a non-constant regular function. We continue the proof by induction on $n$, with the case $n=1$ being trivial.

Let $U^{\prime}$ be the projection of $U$ along the coordinate $x_{n}$. For every point $p^{\prime} \in \mathbb{R}^{n-1} \backslash U^{\prime}$ the Boolean function $\varphi$ for $U^{\prime}$ at $p^{\prime}$ coincides with $E_{n}(\psi)$ where $\psi$ is the Boolean function for $U$ at some point $p \in \mathbb{R}^{n} \backslash U$. Then, by (R2) in Definition 5.1, $\varphi$ is regular. The possibility that $\varphi \equiv 1$ contradicts Lemma 5.8. Then, by the inductive hypothesis, $U^{\prime}$ is semi-monotone. It follows that the intersection

$$
V^{\prime}:=U^{\prime} \cap X_{j_{1}, \sigma_{1}, c_{1}} \cap \cdots \cap X_{j_{k}, \sigma_{k}, c_{k}}
$$

is connected for any $0 \leq k \leq n-1$, any $1 \leq j_{1}<\cdots<j_{k} \leq n-1$, any $\sigma_{1}, \ldots, \sigma_{k}$ in $\{<,=,>\}$, and any $c_{1}, \ldots, c_{k}$. Suppose that the intersection

$$
V:=U \cap X_{j_{1}, \sigma_{1}, c_{1}} \cap \cdots \cap X_{j_{k}, \sigma_{k}, c_{k}}
$$

is not connected. Then, by the Vietoris-Begle theorem, the fibre of the projection along the coordinate $x_{n}$ over some point $p^{\prime}=\left(p_{1}, \ldots, p_{n-1}\right) \in V^{\prime}$ is not connected, i.e., for some $p_{n}, x_{n}, y_{n} \in \mathbb{R}$ we have $\left(p^{\prime}, x_{n}\right),\left(p^{\prime}, y_{n}\right) \in U,\left(p^{\prime}, p_{n}\right) \in \mathbb{R}^{n} \backslash U$, and $x_{n}<$ $p_{n}<y_{n}$. It follows that the Boolean function $\psi$ for $U$ at $\left(p^{\prime}, p_{n-1}\right)$ is $\psi \equiv 1$, which is a contradiction.

It remains to consider the case of the intersection $V \cap X_{n, \sigma_{n}, c_{n}}$ for $\sigma_{n} \in\{<,=,>\}$.

Let $\sigma_{n}$ be $=$. We prove that for a point $p \in \mathbb{R}^{n-1} \backslash\left(U \cap X_{n,=, c_{n}}\right)$, if $U \cap X_{n,=, c_{n}} \neq \emptyset$, then the Boolean function $\varphi$ for $U \cap X_{n,=, c_{n}}$ at $p$ is a non-constant regular function. Since the Boolean function $\psi$ for $U$ at $p$ is non-constant regular, according to Theorem 5.5, the corresponding union $C_{\psi}$ of unit cubes is a PL $n$-ball. Then for the union $C_{\varphi}$ of unit $(n-1)$-cubes we have $C_{\varphi}=C_{\psi+} \cap C_{\psi-}$, otherwise the intersection of $U$ with the cylinder over the corresponding octant in $\mathbb{R}^{n-1}$ centred at $p$ would be disconnected, which is a contradiction. It follows that $C_{\varphi}$ is a PL $(n-1)$-ball, thus $\varphi$ is non-constant regular. 
By the inductive hypothesis, $U \cap X_{n,=, c_{n}}$ is a semi-monotone set, in particular every set $V \cap X_{n,=, c_{n}}$ is connected.

Suppose that some set of the kind $V \cap X_{n, \sigma_{n}, c_{n}}$, where $\sigma_{n} \in\{<,>\}$, were not connected. Since $V$ is connected, the set $V \cap X_{n,=, c_{n}}$ would be disconnected, which is a contradiction.

\section{Appendix: topological background}

Definition 6.1. Let $Z$ be a closed (open) PL ( $n-1)$-ball, $X, Y$ be closed (respectively, open) PL $n$-balls, and

$$
\bar{Z}=\bar{X} \cap \bar{Y}=\partial X \cap \partial Y .
$$

We say that $X \cup Y \cup Z$ is obtained by gluing $X$ and $Y$ along $Z$.

Proposition 6.2 ([4, Lemma 1.10]). Let $X$ and $Y$ be closed PL n-balls and $h: \partial X \rightarrow \partial Y$ a PL homeomorphism. Then $h$ extends to a PL homeomorphism $h_{1}: X \rightarrow Y$.

Proposition 6.3 ([4, Corollary $\left.\left.3.13_{n}\right]\right)$. Let $X$ be a closed PL $n$-ball, $Y$ be a closed $(n+1)$-ball, $\partial Y$ be its boundary (the PL n-sphere), and let $X \subset \partial Y$. Then $\overline{\partial Y \backslash X}$ is a PL n-ball.

Proposition 6.4 ([4, Corollary 3.16]). Let X, Y, Z be closed PL balls as in Definition 6.1, and $X \cup Y$ be obtained by gluing $X$ and $Y$ along $Z$. Then $X \cup Y$ is a closed PL n-ball.

Proposition 6.5 ([5, Lemma I.3.8]). Let $X, Y \subset \mathbb{R}^{n}$ be compact polyhedra such that $X$ and $X \cup Y$ are closed $P L n$-balls. Let $X \cap Y$ be a closed $P L(n-1)$-ball contained in $\partial X$, and suppose the interior of $X \cap Y$ is contained in the interior of $X \cup Y$. Then $Y$ is a closed PL n-ball.

Definition 6.6. An $n$-dimensional shellable cell complex is defined by induction as follows.

1. Any PL regular $n$-cell $A$ is a shellable complex.

2. If $W$ is an $n$-dimensional shellable complex, $B$ is a PL regular $n$-cell, and $C$ is a PL regular $(n-1)$-cell in the boundaries of both $W$ and $B$, then the result of gluing $W$ and $B$ along $C$ is a shellable complex.

Proposition 6.7. Any n-dimensional shellable cell complex is a PL regular $n$-cell.

Proof. Follows from Proposition 6.4 by the induction in Definition 6.6.

Definition 6.8 ([4, Ch. 4]). A pair $\left(Q^{m}, Q^{n}\right)$ of PL manifolds, in particular balls or spheres, is proper if $Q^{n} \cap \partial Q^{m}=\partial Q^{n}$. A proper pair is locally flat if each point $\mathbf{x} \in Q^{n}$ has a neighbourhood in $\left(Q^{m}, Q^{n}\right)$ homeomorphic (as a pair) to an open set in $\left(\mathbb{R}_{+}^{m}, \mathbb{R}_{+}^{n} \times 0\right)$. (It is clear that the pair $\left(\partial Q^{m}, \partial Q^{n}\right)$ is then also locally flat.) The standard ball pair is $\left([-1,1]^{m},[-1,1]^{n} \times 0\right)$, and $\left(\partial[-1,1]^{m}, \partial[-1,1]^{n} \times 0\right)$ is the standard sphere pair. A ball or a sphere pair is unknotted if it is PL homeomorphic to the appropriate standard pair of appropriate dimension. 
Proposition 6.9 (Schönflies theorem, [4, 3.37]). If $n \neq 4$ then any locally flat pair of PL spheres $\left(S^{n}, S^{n-1}\right)$ is unknotted.

The following statement is apparently well-known in PL-topology. However, since we do not have a good reference, we present a proof communicated to us by N. Mnev.

Proposition 6.10. If $n \neq 4,5$, then any locally flat pair of closed PL balls $\left(B^{n}, B^{n-1}\right)$ is unknotted.

Proof. Let $S^{n-1}$ be the boundary of $B^{n}$, and $S^{n-2}$ be the boundary of $B^{n-1}$. Let $B_{+}^{n}$ and $B_{-}^{n}$ be the two parts of $B^{n}$ separated by $B^{n-1}$, and let $S_{+}^{n-1}$ and $S_{-}^{n-1}$ be the corresponding parts of $S^{n-1}$. For $n \neq 5$, Proposition 6.9 implies that the pair $\left(S^{n-1}, S^{n-2}\right)$ of PL spheres is unknotted, and $S_{ \pm}^{n-1}$ are PL $n$-balls.

Let $\left(C^{n}, D^{n-1}\right)$ be the cone pair with base $\left(S^{n-1}, S^{n-2}\right)$. Then $W^{n}:=B^{n} \cup C^{n}$ is a PL $n$-sphere, $V^{n-1}:=B^{n-1} \cup D^{n-1}$ is a PL $(n-1)$-sphere, and the pair $\left(W^{n}, V^{n-1}\right)$ is locally flat. For $n \neq 4$, Proposition 6.9 implies that the pair $\left(W^{n}, V^{n-1}\right)$ of PL spheres is unknotted, and the two parts of $W^{n}$ separated by $V^{n-1}$ are PL $n$-balls. But these two parts are the unions $B_{ \pm}^{n} \cup E_{ \pm}^{n}$, where $E_{+}^{n}$ and $E_{-}^{n}$ are cones over $S_{+}^{n-1}$ and $S_{-}^{n-1}$ respectively. Since $S_{ \pm}^{n-1}$ are PL $n$-balls, Proposition 6.5 implies that so are the $B_{ \pm}^{n}$.

Remark 6.11. Proposition 6.10 is also true in the case $n=5$ but available proofs are more complex, and we do not need this case here.

Proposition 6.12. For $n \leq 4$ any acyclic simplicial subcomplex $X$ of the $n$-simplex $\Delta$ has a vertex with an acyclic link.

Proof. We will consider only the most complex case of $n=4$.

1. If $X$ is one-dimensional, then, being acyclic, $X$ is a tree. Then $X$ has a leaf with acyclic link of a vertex.

2. If $X$ contains a 3-simplex, say $\delta$, then there is the only vertex, say $v$, in $\Delta \backslash \bar{\delta}$. If no simplices apart from $\delta$ and its faces are in $X$, then all vertices of $X$ have acyclic links. Otherwise, $X$ is homotopy equivalent to the suspension of the link of $v$ in $X$, hence the link is acyclic. This covers the cases when $X=\Delta$ and when $\operatorname{dim} X=3$.

3. Suppose that $X$ is two-dimensional. Since any two 2-simplices in $\Delta$ have a common vertex, the one-dimensional part of $X$ consists of trees which cannot have all leaves at some vertices of 2-simplices of $X$ (otherwise $X$ would have a non-trivial 1-cycle). Hence either such a tree has a leaf with its adjacent vertex as its acyclic link, or $X$ is pure twodimensional.

4. Suppose that $X$ is pure two-dimensional.

4(a) There are ten 2-simplices in $\Delta$, and they cannot all be in $X$ since the 2-skeleton of $\Delta$ is not acyclic. Removing one of them, we get the 2-skeleton $Z$ of an acyclic 3dimensional complex consisting of three 3 -simplices. Hence $Z$ has three independent 2 -cycles, and we have to remove at least three 2-simplices to make $Z$ acyclic. It follows that $X$ has at most six 2-simplices.

4(b) If a vertex $v$ of $X$ has one adjacent 2-simplex in $X$ or two adjacent 2-simplices having a common edge, then $v$ has a link in $X$ which is a tree. If $v$ has two adjacent 2simplices in $X$ without a common edge, then $X$, being acyclic, consists of just these two 
2-simplices, and any vertex in $X$ different from $v$ has an acyclic link. It follows that if $X$ does not have any vertices with acyclic links, then for each vertex $v$ of $X$ there should be at least three 2-simplices having $v$ as a common vertex. If $v$ has exactly three adjacent 2simplices, the link $L$ of $v$ is connected, since any disconnected graph with three edges has at least five vertices. If $L$ is not acyclic, then it is a triangle (the boundary of a 2-simplex). Since there are five vertices (otherwise $X$ would be a subcomplex of a three-dimensional simplex), $X$ must have at least five 2-simplices.

It remains to consider the cases of five and six 2-simplices in $X$.

4 (c) Let $X$ have exactly five 2 -simplices. If no vertex has an acyclic link in $X$ (and hence, by 4(b), all links are triangles), then each edge in $X$ is shared by exactly two 2 -simplices. But this is impossible since there are 15 edges to divide into pairs.

4(d) Suppose that $X$ has exactly six 2-simplices. Since the average number of simplices adjacent to the vertices of $X$ is $18 / 5$, there should be a vertex $v$ of $X$ with exactly three adjacent 2-simplices. If the link $L$ of $v$ is not acyclic, then, by 4(b), it is a triangle. Let $w$ be the vertex of $X$ different from $v$ and from the three vertices of $L$. Then $v$ is not in the link $M$ of $w$, hence $M$ (being a subset of $L$ ) is either acyclic or equal to $L$. In the latter case $X$ is combinatorially equivalent to a triangular bipyramid, hence is a non-trivial 2-cycle. This is a contradiction.

Example 6.13. The following example shows that Proposition 6.12 does not hold for $n=5$.

Consider a hexagon with vertices $0, \ldots, 5$.

Attach to it the boundary of a 3-simplex with vertices $0,1,2,3$ without the simplex (013). That is a cell contractible to the union of the hexagon edges (01), (12), (23). Repeat the same construction, replacing $0,1,2,3$ by $2,3,4,5$, and then repeat again, replacing $2,3,4,5$ by $4,5,0,1$.

No two of these three cells have common 2-simplices, and their common edges are all on the hexagon. Hence, the union $Y$ of these cells is contractible to the hexagon and is homotopy equivalent to a circle.

Attach the 2-simplex (135) to $Y$ making the resulting simplicial complex $X:=Y \cup$ (135) contractible, and therefore acyclic. Then the links in $X$ of the vertices $0,2,4$ have cycles of length three, while the links in $X$ of the vertices $1,3,5$ have cycles of length four. Thus $X$ is an acyclic subcomplex of a five-dimensional simplex $\Delta$ having no vertices with acyclic links.

Proposition 6.14 ([7, Ch. 8, (2.14)]). Let $X \subset \mathbb{R}^{m+n}$ be a definable set, and let $\pi$ : $\mathbb{R}^{m+n} \rightarrow \mathbb{R}^{m}$ be the projection map. Then there exist an integer $N>0$ and a definable (not necessarily continuous) map $f: X \rightarrow \Delta$, where $\Delta$ is an $(N-1)$-simplex, such that for every $\mathbf{x} \in \mathbb{R}^{m}$ the restriction $f_{\mathbf{x}}: X \cap \pi^{-1}(\mathbf{x}) \rightarrow \Delta$ of $f$ to $X \cap \pi^{-1}(\mathbf{x})$ is a definable homeomorphism onto a union of faces of $\Delta$.

Corollary 6.15. Using the notations from Proposition 6.14, suppose that all fibres $X \cap$ $\pi^{-1}(\mathbf{x})$ are definable compact sets. Then there is a partition of $\pi(X)$ into a finite number of definable sets $T \subset \mathbb{R}^{m}$ such that all fibres $X \cap \pi^{-1}(\mathbf{x})$ with $\mathbf{x} \in T$ are definably homeomorphic to the same simplicial complex. 
Proof. There are a finite number of different unions of faces in $\Delta$. Since $f$ is definable, the pre-image of any such union under the map $f \circ \pi^{-1}$ is a definable set.

Acknowledgments. We thank S. Ferry, J. McClure, and N. Mnev for useful discussions. S. Basu was supported in part by NSF grant CCF-0915954, A. Gabrielov was supported in part by NSF grant DMS-0801050.

\section{References}

[1] Bochnak, J., Coste, M., Roy, M.-F.: Real Algebraic Geometry. Springer, Berlin (1998) Zbl 0912.14023 MR 1659509

[2] Coste, M.: An Introduction to o-minimal Geometry. Dip. Mat. Univ. Pisa, Dottorato di Ricerca in Matematica, Istituti Editoriale e Poligrafici Internazionali, Pisa (2000)

[3] Gabrielov A., Vorobjov, N.: Approximation of definable sets by compact families, and upper bounds on homotopy and homology. J. London Math. Soc. 80, 35-54 (2009) Zbl 1177.14097 MR 2520376

[4] Rourke, C. P., Sanderson, B. J.: Introduction to Piecewise-Linear Topology. Springer, Berlin (1972) Zbl 0254.57010 MR 0350744

[5] Shiota, M.: Geometry of Subanalytic and Semialgebraic Sets. Birkhäuser, Basel (1997) Zbl 0889.32006 MR 1463945

[6] Spanier, E. H.: Algebraic Topology. Springer, Berlin (1966) Z Zbl 0145.43303 MR 0210112

[7] van den Dries, L.: Tame Topology and o-minimal Structures. Cambridge Univ. Press, Cambridge (1998) Zbl 0953.03045 MR 1633348 